\title{
Psicobióticos: potenciais alternativas aos ansiolíticos convencionais?
}

\author{
Psychobiotics: potential alternatives to conventional anxiolytics? \\ Psicobióticos: ¿alternativas potenciales a los ansiolíticos convencionales?
}

\author{
Karoline Cristina Jatobá da Silva \\ ORCID: https://orcid.org/0000-0002-2797-7407 \\ Universidade Federal de Alagoas, Brasil \\ E-mail: karol.jatobas@gmail.com \\ Maria Gabryelle Jatobá Pereira de Brito \\ ORCID: https://orcid.org/0000-0001-7395-6933 \\ Universidade Federal de Pernambuco, Brasil \\ E-mail: gabryelle.brito@ufpe.br \\ Alyne Almeida de Lima \\ ORCID: https://orcid.org/0000-0002-6174-7402 \\ FIOCRUZ- Instituto Gonçalo Moniz, Brasil \\ E-mail: prof.alynealmeida@gmail.com \\ Max Denisson Maurício Viana \\ ORCID: https://orcid.org/0000-0002-1650-4460 \\ Universidade Federal da Bahia, Brasil \\ E-mail: max.viana@ufba.br
}

\begin{abstract}
Resumo
Compreendendo que nas últimas décadas os psicobióticos, uma nova classe de probióticos, direcionaram muitos pesquisadores a se concentrarem no estudo dos mesmos em relação ao seu potencial terapêutico para transtornos psíquicos, o presente estudo compilou dados da literatura científica a respeito das recentes descobertas relacionadas aos psicobióticos como uma potencial alternativa para o tratamento da ansiedade. Para tal foi realizada uma revisão integrativa de literatura, por meio de consultas a artigos publicados nos últimos seis anos por meio das bases de dados PubMed/MEDLINE e SciELO, utilizando os seguintes descritores:"Probiotics and anxiety", "Psycobiotcs and anxiety", publicados na íntegra em inglês. Totalizando 267 estudos encontrados, dos quais meramente 14 artigos foram elegíveis. A princípio foram discutidos os benefícios gerados pelos psicobióticos para a saúde mental, bem como sua importância na compreensão dos mecanismos envolvidos na relação do eixo cérebro-microbioma-intestino quanto aos transtornos psicológicos, especialmente a ansiedade. Por meio da análise dos estudos pré-clínicos e clínicos selecionados, foi possível identificar cepas de probióticos que apresentam maior potencial a candidatos psicobióticos, com ênfase no tratamento da ansiedade. De forma geral, os estudos demonstraram a eficácia dos psicobióticos, os quais foram capazes de atenuar os sintomas causados pelos quadros de ansiedade, melhorando funções do sistema nervoso central, com baixa ocorrência ou ausência de efeitos adversos. Assim, futuramente podem compor uma nova abordagem terapêutica para o tratamento dos sintomas de ansiedade e outras comorbidades psiquiátricas.
\end{abstract}

Palavras-chave: Probióticos; Ansiedade; Psicofarmacologia.

\begin{abstract}
Understanding that in the last decades psychobiotics, a new class of probiotics, have directed many researchers to focus on studying them in relation to their therapeutic potential for psychic disorders, the present study compiled data from the scientific literature regarding recent discoveries related to psychobiotics as a potential alternative for the treatment of anxiety. For this purpose, an integrative literature review was carried out, through consultations to articles published in the last six years through the PubMed/MEDLINE and SciELO databases, using the following descriptors: "Probiotics and anxiety","Psycobiotcs and anxiety", published in full in English. A total of 267 studies were found, of which merely 14 articles were eligible. At first, the benefits generated by psychobiotics for mental health were discussed, as well as their importance in understanding of the mechanisms involved in the brain-microbiome- intestine axis relationship for psychological disorders, especially anxiety. Through the analysis of selected pre-clinical and clinical studies, it was possible to identify strains of probiotics that have greater potential for psychobiotic candidates, with an emphasis on the treatment of anxiety. In general, studies have shown the effectiveness of psychobiotics, which were able to mitigate the symptoms caused by anxiety disorders, improving central nervous system functions, with low occurrence or absence of adverse effects. Thus, in the future, they can compose a new therapeutic approach for the treatment of anxiety symptoms and other psychiatric comorbidities.
\end{abstract}

Keywords: Probiotics; Anxiety; Psychopharmacology. 


\section{Resumen}

Entendiendo que en las últimas décadas los psicobióticos, una nueva clase de probióticos, handirigido a muchos investigadores a centrarse en estudiarlos en relación con su potencial terapéutico para los trastornos psíquicos, el presente estudio recopiló datos de la literatura científica sobre descubrimientos recientes relacionados con los psicobióticos como alternativa potencial para el tratamiento de la ansiedad. Para ello, se realizó una revisión integradora de la literatura, mediante consultas a artículos publicados en los últimos seis años através de las bases de datos PubMed/MEDLINE y SciELO, utilizando los siguientes descriptores: "Probióticos y ansiedad","Psicobióticos y ansiedad", publicados íntegramente en inglés. Se encontraron un total de 267 estudios, de los cuales solo fueron elegibles 14 artículos. En un principio se discutieron los beneficios que generan los psicobióticos para la salud mental, así como sus importancia en la comprensión de los mecanismos involucrados en la relación cerebro-microbioma- eje intestino para los trastornos psicológicos, especialmente la ansiedad.Através del análisis de estudios preclínicos y clínicos seleccionados, fue posible identificar cepas de probióticos que tienen mayor potencial para candidatos psicobióticos, con énfasis en el tratamiento de la ansiedad. En general, los estudios han demostrado la efectividad de los psicobióticos, que fueron capaces de mitigar los síntomas provocados por los trastornos de ansiedad, mejorando las funciones del sistema nervioso central, con baja ocurrencia o ausencia de efectos adversos. Entonces, en el futuro, pueden componer un nuevo enfoque terapéutico para el tratamiento de los síntomas de ansiedad y otras comorbilidades psiquiátricas.

Palabras clave: Probióticos; Ansiedad; Psicofarmacología.

\section{Introdução}

Os transtornos de ansiedade se configuram como os transtornos mentais de mais alta prevalência e debilidade nas populações em estudo. A ansiedade é caracterizada por medo e desespero persistentes, acompanhada pelo desenvolvimento de sintomas físicos como taquicardia, tensão, dor e incapacidade de relaxar, resultando em prejuízos físicos e cognitivos que impactam diretamente a qualidade de vida em todas as esferas (Yang et al., 2021).

A prevalência deste transtorno mental tem aumentado significativamente principalmente em países de média e baixa renda. Estima-se que cerca 264 milhões de pessoas no mundo sejam acometidas por algum transtorno de ansiedade. Segundo a Organização Mundial da Saúde (OMS), o Brasil lidera a prevalência de casos, atingindo aproximadamente 9,3\% da população, superior, por exemplo, aos casos de depressão, no qual o país ocupa o quinto lugar (5,8\%) (WHO, 2017). É importante salientar que em muitos casos as pessoas vivenciam depressão maior e ansiedade simultaneamente.

Atualmente, a associação de psicoterapia e farmacoterapia é a orientação mais eficaz no que se refere ao tratamento para transtornos de ansiedade. Dentre as classes mais prescritas, incluem-se: inibidores seletivos da recaptação da serotonina ou serotonina-norepinefrina, seguidos dos benzodiazepínicos. Outras opções de tratamento incluem ainda: pregabalina, antidepressivos tricíclicos, ou buspirona (Bandelow, Michaelis \& Wedekind, 2017). Entretanto, o risco de efeitos colaterais, tais como: tolerância, dependência, síndrome de abstinência, dentre outros, têm levado pacientes com necessidade de tratamento a buscar alternativas terapêuticas menos prejudiciais (Mangolini et al., 2019).

Alternativamente, nos últimos anos, evidências crescentes destacaram uma ligação entre a microbiota intestinal e transtornos neurológicos e psiquiátricos (Gualtieri et al., 2020). Nesse contexto, estudos relataram que algumas cepas de bactérias (psicobióticas) atuariam em nível intestinal modulando vias inflamatórias, hormonais ou neurais, resultando em uma melhora dos sintomas de ansiedade. Os psicobióticos são um grupo de probióticos que afetam as funções e comportamentos relacionados ao sistema nervoso central (SNC) mediados pelo eixo intestino-cérebro através de vias imunológicas, humorais, neurais e metabólicas para melhorar não apenas a função gastrointestinal (GI) mas também os sintomas de ansiedade (Cheng et al., 2019).

Além das modalidades terapêuticas que possuem, os psicobióticos são de grande interesse por serem potencialmente livres de efeitos colaterais cognitivos e das propriedades dependógenas dos tratamentos convencionais (Liu, Walsh \& Sheehan, 2019). Diante disso, a presente revisão visou abordar as recentes descobertas relacionadas ao emprego dos psicobióticos como alternativas potenciais no tratamento da ansiedade. 


\section{Metodologia}

Trata-se de um estudo de caráter exploratório, descritivo, de natureza qualitativa, com coleta retrospectiva de dados, utilizando-se para isto da revisão integrativa da literatura através da pesquisa bibliográfica de artigos científicos em bases de dados online, a fim de concretizar o objetivo proposto. Tal método foi selecionado para a pesquisa por proporcionar uma síntese do conhecimento e possibilitar a aplicação dos resultados de pesquisas significativas na prática (Souza, Silva \& Carvalho, 2010).

Foram estabelecidos critérios de busca, critérios de inclusão e exclusão para a seleção da amostra, para posterior organização e apresentação dos resultados e conclusões. As buscas foram realizadas na base de dados: PubMed/MEDLINE e SciELO através dos seguintes descritores: "Probiotics and anxiety", "Psycobiotcs and anxiety", entre os meses de fevereiro e março de 2021.

Foram considerados elegíveis: artigos de livre acesso, completos, publicados a partir do ano 2015, redigidos na língua inglesa e que tivessem relacionados com o tema proposto; excluindo-se, portanto, aqueles que não contemplassem tais critérios.

Em seguida, foram avaliados os títulos e os resumos dos artigos a fim de verificar se os mesmos estavam de acordo com os objetivos do estudo. Em caso de divergências ou dúvidas o artigo era lido na íntegra para definição e posterior composição do estudo.

\section{Resultados}

Ao todo foram encontrados 267 artigos, dos quais 231 foram relacionados à "probióticos e ansiedade" e 36 relacionados à "psicobióticos e ansiedade". Desses, foram excluídos 262 artigos por não atenderem aos critérios de inclusão e apenas 14 foram incluídos na revisão integrativa.

É importante salientar que apesar de identificados muitos estudos, poucos estavam relacionados ao tema proposto, indicando que o assunto ainda é pouco abordado em pesquisas acadêmicas.

O Quadro 1 identifica os 14 artigos utilizados na revisão integrativa, destacando: Autor/ano, título do artigo e objetivo e conclusão do estudo. 
Quadro 1 - Caracterização dos estudos quanto aos seus objetivos e principais achados.

\begin{tabular}{|c|c|c|c|}
\hline Autor/Ano & Título & Objetivo & Conclusão \\
\hline $\begin{array}{l}\text { Peirce, J. M., } \\
\text { Alviña, K. } \\
\text { (2019) }\end{array}$ & $\begin{array}{l}\text { The role of inflammation and } \\
\text { the gut microbiome in } \\
\text { depression and anxiety }\end{array}$ & $\begin{array}{l}\text { Discutir mecanismos específicos pelos quais o microbioma } \\
\text { intestinal pode promover o desenvolvimento de transtornos } \\
\text { mentais, como depressão e ansiedade. }\end{array}$ & $\begin{array}{l}\text { Os resultados mostraram que muitos transtornos } \\
\text { mentais, como ansiedade, podem se originar da } \\
\text { exposição precoce ao estresse e à inflamação. }\end{array}$ \\
\hline $\begin{array}{l}\text { Butler, M. I. } \\
\text { et al. } \\
\text { (2019) }\end{array}$ & $\begin{array}{l}\text { The Gut Microbiome and } \\
\text { Mental Health: WhatShould } \\
\text { We Tell Our Patients?: Le } \\
\text { microbiote Intestinal et la } \\
\text { Santé Mentale : que Devrions- } \\
\text { Nous dire à nos Patients? }\end{array}$ & $\begin{array}{l}\text { Fornecer uma visão geral do microbioma intestinal, e da } \\
\text { relação do eixo cérebro-intestino e função psicológica. } \\
\text { Descrever os princípios fundamentais e as técnicas básicas } \\
\text { usadas na pesquisa do eixo microbioma-intestino-cérebro. }\end{array}$ & $\begin{array}{l}\text { Os resultados evidenciaram que algumas espécies } \\
\text { de bactérias dos gêneros } \\
\text { Lactobacillus e Bifidobacterium demonstraram } \\
\text { ter efeitos psicobióticos, melhorando os sintomas } \\
\text { de ansiedade de voluntários saudáveis com } \\
\text { baixos escores de humor basais. }\end{array}$ \\
\hline $\begin{array}{l}\text { Steenbergen, L. } \\
\text { et al. } \\
\text { (2015) }\end{array}$ & $\begin{array}{l}\text { A randomized controlled trial } \\
\text { to test the effect of } \\
\text { multispecies probiotics on } \\
\text { cognitive reactivity to sad } \\
\text { mood }\end{array}$ & $\begin{array}{c}\text { Avaliar o pool: Bifidobacterium } \\
\text { bifidum W23, Bifidobacterium lactis W52, Lactobacillus } \\
\text { acidophilus W37, Lactobacillus brevis W63, Lactobacillus } \\
\text { casei W56, Lactobacillus salivariusS24 e Lactococcus } \\
\text { lactis (S19 e S58) na reatividade cognitiva no transtorno de } \\
\text { ansiedade, em indivíduos não deprimidos. }\end{array}$ & $\begin{array}{l}\text { Os resultados forneceram a primeira evidência de } \\
\text { que a ingestão de probióticos pode ajudar a } \\
\text { reduzir pensamentos negativos associados ao } \\
\text { humor triste, mitigando sintomas de ansiedade. }\end{array}$ \\
\hline $\begin{array}{l}\text { Liu, Y,W. } \\
\text { et al. } \\
\text { (2018) }\end{array}$ & $\begin{array}{l}\text { Psychotropic effects of } \\
\text { lactobacillus } \\
\text { plantarumps } 128 \text { in early } \\
\text { life-stressed and naïve } \\
\text { adult mice }\end{array}$ & $\begin{array}{l}\text { Examinar os efeitos psicotrópicos de uma potencial } \\
\text { bactéria psicobiótica, Lactobacillus plantarum cepa PS128 } \\
\text { (PS128), para melhora da ansiedade em roedores. }\end{array}$ & $\begin{array}{l}\text { Os resultados sugerem que a ingestão crônica de } \\
\text { PS128 pode melhorar comportamentos } \\
\text { semelhantes aos da ansiedade e da depressão e } \\
\text { modular os neuroquímicos relacionados aos } \\
\text { transtornos afetivos. Assim, a cepa PS128 } \\
\text { demonstra propriedades psicotrópicas e tem } \\
\text { grande potencial para melhorar os sintomas } \\
\text { relacionados ao estresse e à ansiedade. }\end{array}$ \\
\hline $\begin{array}{l}\text { Hao, Z., Wang, W., Guo, R., \& } \\
\text { Liu, H. } \\
\text { et al. } \\
\text { (2019) }\end{array}$ & $\begin{array}{l}\text { Faecalibacterium prausnitzii } \\
\text { (ATCC 27766) has preventive } \\
\text { and therapeutic effects on } \\
\text { chronic unpredictable mild } \\
\text { stress-induced depression-like } \\
\text { and anxiety-like behavior in } \\
\text { rats. }\end{array}$ & $\begin{array}{l}\text { Testar o potencial psicobiótico Faecalibacterium } \\
\text { prausnitzii (ATCC 27766) quanto aos efeitos ansiolíticos e } \\
\text { antidepressivos e à reversão do impacto do estresse crônico } \\
\text { imprevisível leve em ratos. }\end{array}$ & $\begin{array}{l}\text { Os resultados obtidos no presente estudo } \\
\text { forneceram evidências adicionais de que a } \\
\text { administração de } F \text {. prausnitzii possui efeitos } \\
\text { ansiolíticos e antidepressivos desempenhando um } \\
\text { papel importante, mediado pelo eixo intestino- } \\
\text { cérebro. }\end{array}$ \\
\hline
\end{tabular}




\begin{tabular}{|c|c|c|c|}
\hline $\begin{array}{l}\text { Yunes, R. A. } \\
\text { et al. } \\
\text { (2020) }\end{array}$ & $\begin{array}{l}\text { A Multi-strain Potencial } \\
\text { Probiotic Formulation GABA- } \\
\text { Producing Lactobacillus } \\
\text { plantarum } 90 \mathrm{ks} \text { and } \\
\text { Bifidobacterium adolecentis } \\
150 \text { with Antidepressant } \\
\text { Effects. }\end{array}$ & $\begin{array}{c}\text { Avaliar as propriedades probióticas das cepas selecionadas, } \\
\text { bem como seus efeitos ansiolíticos e antidepressivos em } \\
\text { camundongos. }\end{array}$ & $\begin{array}{l}\text { Os resultados estabeleceram que a ingestão da } \\
\text { composição probiótica baseada nas cepas } \\
\text { selecionadas por camundongos BALB/c por } 2 \\
\text { semanas reduziu o comportamento do tipo } \\
\text { ansioso e depressivo no teste de natação forçada, } \\
\text { semelhantemente ao grupo tratado com } \\
\text { fluoxetina. }\end{array}$ \\
\hline $\begin{array}{l}\text { Barros-Santos, T. } \\
\text { et al. } \\
\text { (2020) }\end{array}$ & $\begin{array}{l}\text { Effects of chronic treatment } \\
\text { with new strains of } \\
\text { Lactobacillus plantarum on } \\
\text { cognitive, anxiety- and } \\
\text { depressive-like behaviors in } \\
\text { male mice. }\end{array}$ & $\begin{array}{l}\text { Avaliar os efeitos da administração crônica de duas cepas } \\
\text { de Lactobacillus plantarum (Lp } 286 \text { e Lp 81) isoladas da } \\
\text { fermentação de cacau (Theobroma cacao L.) e cupuaçu } \\
\text { (Theobroma grandiflorum), respectivamente, em } \\
\text { comportamentos cognitivos, ansiosos e depressivos em } \\
\text { camundongos. }\end{array}$ & $\begin{array}{l}\text { Os achados indicam que o tratamento com } \\
\text { Lactobacillus plantarum } 286 \text { induziu efeitos do } \\
\text { tipo ansiolítico, possivelmente aumentando os } \\
\text { níveis de GABA no trato GI e, } \\
\text { consequentemente, no SNC. }\end{array}$ \\
\hline $\begin{array}{l}\text { Reis, D. J. } \\
\quad \text { et al. } \\
(2018)\end{array}$ & $\begin{array}{l}\text { The anxiolytic effect of } \\
\text { probiotics: A systematic } \\
\text { review and meta-analysis of } \\
\text { the clinical and preclinical } \\
\text { literature }\end{array}$ & $\begin{array}{l}\text { Avaliar as evidências clínicas e pré-clínicas sobre o efeito } \\
\text { da administração de probióticos na ansiedade. }\end{array}$ & $\begin{array}{l}\text { O estudo demonstrou que a administração de } \\
\text { probióticos reduziu o comportamento de } \\
\text { ansiedade em roedores. Ademais, identificou } \\
\text { Lactobacillus rhamnosus como uma espécie } \\
\text { psicobiótica ansiolítica candidata por estudos } \\
\text { pré-clínicos e clínicos. }\end{array}$ \\
\hline $\begin{array}{l}\text { Colica, C. } \\
\text { et al } \\
(2017)\end{array}$ & $\begin{array}{l}\text { Evidences of a New } \\
\text { Psychobiotic Formulation on } \\
\text { Body Composition and } \\
\text { Anxiety }\end{array}$ & $\begin{array}{l}\text { Avaliar os efeitos de uma nova formulação psicobiótica no } \\
\text { tratamento da ansiedade. }\end{array}$ & $\begin{array}{c}\text { A ingestão de psicobióticos selecionados por três } \\
\text { semanas representou importante abordagem na } \\
\text { resolução de problemas relacionados à obesidade } \\
\text { e aos distúrbios de comportamento relacionados à } \\
\text { ansiedade. }\end{array}$ \\
\hline $\begin{array}{l}\text { De Lorenzo A. } \\
\text { et al. } \\
\text { (2017) }\end{array}$ & $\begin{array}{l}\text { Can psychobiotics intake } \\
\text { modulate psychological } \\
\text { profile and body composition } \\
\text { of women affected by normal } \\
\text { weight obese syndrome and } \\
\text { obesity? A double blind } \\
\text { randomized clinical Trial }\end{array}$ & $\begin{array}{c}\text { Investigar se a ingestão crônica de probióticos poderia } \\
\text { modular o estado psicológico, o comportamento alimentar } \\
\text { e a composição corporal de obesos com peso normal e pré- } \\
\text { obesos em comparação com mulheres magras com peso } \\
\text { normal. }\end{array}$ & $\begin{array}{l}\text { Os resultados destacaram que a ingestão por } 3 \\
\text { semanas da nova formulação da suspensão oral } \\
\text { probiótica pode ter potencial como estratégia } \\
\text { terapêutica para prevenção e/ou tratamento de } \\
\text { determinados transtornos de comportamento } \\
\text { alimentar e ansiosos. }\end{array}$ \\
\hline
\end{tabular}




\begin{tabular}{|c|c|c|c|}
\hline $\begin{array}{l}\text { Herman A. } \\
\text { (2019) }\end{array}$ & $\begin{array}{l}\text { Probiotics supplementation in } \\
\text { prophylaxis and treatment of } \\
\text { depressive and anxiety } \\
\text { disorders - a review of current } \\
\text { research. }\end{array}$ & $\begin{array}{c}\text { Discutir a relação entre a composição da microbiota } \\
\text { intestinal e as funções efetivas do SNC e sobre os efeitos } \\
\text { da suplementação de probióticos nos sintomas depressivos } \\
\text { e ansiosos em humanos. }\end{array}$ & $\begin{array}{l}\text { O uso de probióticos pode prevenir distúrbios na } \\
\text { composição da microbiota intestinal como } \\
\text { resultado do estresse crônico, ao reduzir a } \\
\text { inflamação e aumentar a biossíntese de } \\
\text { serotonina. Sendo assim, psicobióticos, } \\
\text { futuramente, poderão ser usados na profilaxia, } \\
\text { bem como compor o tratamento de transtornos } \\
\text { depressivos e de ansiedade. }\end{array}$ \\
\hline $\begin{array}{l}\text { Gualtieri, P. } \\
\text { et al. } \\
\text { (2020) }\end{array}$ & $\begin{array}{l}\text { Psychobiotics Regulate the } \\
\text { Anxiety Symptoms in Carriers } \\
\text { of Allele A of IL-1 } \beta \text { Gene: A } \\
\text { Randomized, Placebo } \\
\text { Controlled Clinical Trial. }\end{array}$ & $\begin{array}{l}\text { Avaliar o efeito combinado do polimorfismo IL-1 } \beta \text { e da } \\
\text { administração de probióticos em fenótipos de transtorno de } \\
\text { humor na população italiana. }\end{array}$ & $\begin{array}{l}\text { O consumo de psicobióticos atenua os sintomas } \\
\text { de ansiedade, especialmente em adultos } \\
\text { saudáveis com o alelo A menor de rs } 16944 \text { como } \\
\text { fator de risco. Os resultados encorajam o uso de } \\
\text { probióticos em transtornos de ansiedade e } \\
\text { sugerem estudos de associação genética para } \\
\text { terapia personalizada com psicobióticos. }\end{array}$ \\
\hline $\begin{array}{l}\text { Smith, K. S. } \\
\text { et al. } \\
\text { (2019) }\end{array}$ & $\begin{array}{l}\text { Psychobiotics as treatment for } \\
\text { anxiety, depression, and } \\
\text { related symptoms: a } \\
\text { systematic review }\end{array}$ & $\begin{array}{l}\text { Avaliar a eficácia dos probióticos, prebióticos ou } \\
\text { simbióticos como um potencial tratamento para sintomas } \\
\text { de depressão, ansiedade e estresse (como psicobióticos). }\end{array}$ & $\begin{array}{l}\text { Os resultados demonstraram que o uso de } \\
\text { probióticos e prebióticos reduziram os escores de } \\
\text { ansiedade em adultos com síndrome da fadiga } \\
\text { crônica e câncer de laringe em condições pré- } \\
\text { operatórias, oferecendo um potencial tratamento } \\
\text { alternativo em transtornos de humor como a } \\
\text { ansiedade, bem como dos sintomas que os } \\
\text { acompanham. }\end{array}$ \\
\hline $\begin{array}{l}\text { Liu, R. T. } \\
\quad \text { et al } \\
(2019)\end{array}$ & $\begin{array}{l}\text { Prebiotics and probiotcs for } \\
\text { depression and anxiety: A } \\
\text { systematic review and } \\
\text { metaanalysis of cotrolled } \\
\text { clinical trials }\end{array}$ & $\begin{array}{l}\text { Avaliar os efeitos de prebióticos e probióticos na depressão } \\
\text { e ansiedade. }\end{array}$ & $\begin{array}{c}\text { Foi observado suporte geral para a eficácia dos } \\
\text { efeitos antidepressivos e ansiolíticos dos } \\
\text { probióticos, mas os efeitos combinados foram } \\
\text { reduzidos pela escassez de ensaios com amostras } \\
\text { clínicas. Portanto, ensaios clínicos randomizados } \\
\text { adicionais com amostras psiquiátricas são } \\
\text { necessários para avaliar totalmente seu potencial } \\
\text { terapêutico. }\end{array}$ \\
\hline
\end{tabular}

Fonte: Autores (2021). 


\section{Discussão}

Os psicobióticos consistem numa classe especial de probióticos, que oferecem benefícios para a saúde mental. Diferem dos probióticos convencionais em sua capacidade de produzir ou estimular a produção de neurotransmissores, ácidos graxos de cadeia curta, hormônios neuroendócrinos e citocinas anti-inflamatórias, cujo espectro de aplicações varia desde o alívio do humor e do estresse até um adjuvante, de fato, no tratamento terapêutico para vários distúrbios do neurodesenvolvimento e neurodegenerativos (Sharma et al., 2021).

Segundo Peirce \& Alviña (2019), o estudo do eixo microbioma intestinal-cérebro está avançando sobre a compreensão dos mecanismos subjacentes aos distúrbios do SNC, tornando claro o papel desse microambiente na modulação da saúde mental.

Isso ressalta o interesse da comunidade científica para realização de estudos que descrevem seus benefícios em relação aos tratamentos com ansiolíticos convencionais, utilizados na prática clínica atual. Nos últimos seis anos, pesquisas emergiram nesse enfoque demonstrando que algumas cepas de psicobióticos resultam em uma melhora dos sintomas de ansiedade e transtornos de humor (Cheng et al., 2019).

Butler et al. (2019) apontaram inclusive que a maioria das espécies que demonstraram tais efeitos provém de dois gêneros: Lactobacillus e Bifidobacterium. No entanto, existem muitas linhagens diferentes dentro desses dois gêneros com efeitos centrais distintos, assim como outros gêneros com atividades símiles ainda não elucidados. Os achados mais recentes, dentro do filtro de seleção desse estudo, estão contemplados no Quadro 1 e serão discutidos a partir de então.

Steenbergen et al. (2015) forneceram, através de um ensaio clínico randomizado com um pool de psicobióticos, a primeira evidência de que a ingestão dessa suplementação poderia auxiliar na redução de pensamentos negativos associados ao humor deprimido, cotando-a como uma importante estratégia preventiva para condições psiquiátricas.

Baseadas nesses resultados, pesquisas pré-clínicas também foram paralelamente sendo realizadas, demonstrando novas cepas e comprovando os efeitos em modelos animais, embasando para posteriormente serem empregadas em maiores ensaios clínicos.

Liu et al. (2016) avaliaram os efeitos de uma potencial cepa psicobiótica, Lactobacillus plantarum cepa PS128 (PS128), em roedores submetidos ou não a estresse precoce. Observaram que a administração da espécie reduziu comportamentos semelhantes aos da ansiedade e depressão bem como os níveis de corticosterona sérica em condições basais e estressadas.

Já no estudo realizado por Hao, Wang, Guo \& Liu (2019), constatou-se a potencialidade da espécie Faecalibacterium prausnitzii (ATCC 27766), a qual é considerada um probiótico de próxima geração com potencial ansiolítico, podendo modificar marcadamente o comportamento e a atividade do eixo hipotálamo-pituitária-adrenal (HPA) relevantes para a depressão e ansiedade. Tais experimentos indicaram o potencial psicobiótico da Faecalibacterium prausnitzii ao induzir nos roedores efeitos preventivos e terapêuticos nos comportamentos de ansiedade e depressão, revertendo o impacto do estresse crônico imprevisível leve, fornecendo evidências adicionais de que a microbiota intestinal desempenha um papel importante, provavelmente mediado pelo eixo intestino-cérebro.

De acordo com as observações de Yunes et al. (2020), a coingestão das cepas L. plantarum 90sk e B. adolescentis 150 reduziu significativamente parâmetros comportamentais que mimetizam estados de ansiedade e depressão em camundongos, de modo semelhante ao fármaco padrão, fluoxetina. Os autores atribuíram atividade ao fato destas cepas estarem relacionadas à síntese de ácido gama-aminobutírico (GABA), principal neurotransmissor inibitório do SNC.

Um estudo com as cepas Lactobacillus plantarum 286 e Lactobacillus plantarum 81, ambas extraídas a partir da fermentação de cacau (Theobroma cacao L.), demonstrou que apenas a primeira induziu efeitos ansiolíticos sem 
comprometimento psicomotor e cognitivo. Esses achados se somam ao conjunto de evidências que sugerem que os psicobióticos podem, de fato, ser incluídos em estudos clínicos como adjuvantes para o tratamento de transtornos psiquiátricos, especialmente aqueles relacionados à ansiedade (Barros-Santos et al., 2020).

Tais evidências pré-clínicas e clínicas corroboram a análise sistemática e de metanálise de Reis, Ilardi \& Punt (2018) sobre o efeito da administração de psicobióticos na ansiedade, destacando-se, neste caso, a espécie Lactobacillus rhamnosus como promissora candidata a estudos clínicos, fomentando a necessidade de maiores investigações neste campo, tendo em vista os resultados promissores apenas em modelos experimentais em animais.

Na clínica, é importante ressaltar que os transtornos de ansiedade estão associados ao aumento de risco para doenças cardíacas coronarianas, consumo abusivo de álcool e de outras substâncias danosas (Reis et al., 2018), além de poder influenciar os comportamentos alimentares, implicando nos fatores psicológicos multifatoriais da obesidade. Para este último, a qual vem sendo associada a um risco aumentado para alguns transtornos mentais, particularmente entre mulheres, "o comer" se torna um mecanismo de enfrentamento para aliviar e lidar com o estresse e as emoções (He et al., 2021).

Nesse contexto, Colica et al. (2017), em estudo clínico, demonstraram que a ingestão durante três semanas, de um pool de psicobióticos composto pelas seguintes bactérias: Streptococcus thermophilus (cepa CNCM número I-1630), Lactobacillus bulgaricus (cepa CNCM números I-1632 e I-1519), Lactococcus lactis subsp. lactis (cepa CNCM número I1631); Lactobacillus acidophilus; Lactobacillus plantarum; Bifidobacterium lactis (CNCM I-2494); Lactobacillus reuteri (DSM 17938), apresentou importante abordagem para solucionar problemas relacionados aos distúrbios de comportamento ligados à ansiedade no que se refere à redução da ansiedade e do apetite relacionado. No entanto, os autores sugerem que novos ensaios clínicos precisam ser realizados em uma população maior e por um período mais longo de tratamento antes que conclusões definitivas possam ser feitas.

Similares aos dados De Lorenzo et al. (2017), um ensaio clínico duplo-cego randomizado que avaliou se a ingestão de um pool de psicobióticos, durante três semanas poderia modular o perfil psicológico e a composição corporal de mulheres normopeso ou obesas. O pool era composto pelas seguintes cepas: Streptococcus thermophilus SGSt01 , Bifidobacterium animalis subsp. Lactis SGB06, Streptococcus thermophillus, Bifidobacterium bifidum SGB02, Lactobacillus delbrueckii spp. Bulgaricus DSM 20081, Lactococcus lactis subsp. Lactis SGLc01, Lactobacillus acidophilus SGL11, Lactobacillus plantarum SGL07, Lactobacillus reuteri SGL01. A ingestão de tais probióticos selecionados, por três semanas, modulou a composição corporal, contaminação bacteriana, pontuações psicopatológicas e comportamento alimentar de mulheres obesas, oferecendo uma abordagem tratável para problemas relacionados à obesidade, estado psicológico e alimentação não saudável.

Em outra perspectiva, Herman (2019) demonstrou a possibilidade do uso profilático de psicobióticos nos distúrbios da composição da microbiota intestinal resultante do estresse crônico. A autora atribui à redução da inflamação e ao aumento da biossíntese de serotonina; isto é, os psicobióticos podem contribuir na prevenção da ansiedade, dada a vantagem principal, como mostram os estudos atuais, que nenhum efeito colateral grave foi observado após sua ingestão.

Vale relembrar que a ansiedade excessiva/suprafisiológica provoca alterações intensas no comportamento, associadas a preocupações generalizadas, acompanhadas ou não por desconforto gastrointestinal, respiratório e tensões musculares afetando a rotina dos indivíduos, em todas as esferas (Minayo, Miranda \& Telhado, 2020).

Este fato remete ao estudo conduzido por Gualtieri et al. (2020), no qual analisaram o efeito combinado do polimorfismo IL-1 $\beta$ e da administração de psicobióticos, por 12 semanas, em fenótipos de transtorno de ansiedade e humor na população italiana. Os pesquisares sugeriram uma redução dos sintomas de ansiedade, especialmente em adultos saudáveis com o alelo A menor de rs16944 como fator de risco, restaurando o equilíbrio psicológico, sem manifestação de eventos adversos. 
Um levantamento realizado por Smith et al. (2019) identificou que a administração de cepas de psicobióticos associadas a prebióticos reduziram os escores de ansiedade em adultos com Síndrome da Fadiga Crônica, de pacientes oncológicos em condições pré-cirúrgicas e reduziram os sintomas depressivos de pacientes de graus leve a moderada. Além disso, pontuaram que o estresse e as respostas imunológicas foram atenuadas após intervenção psicobiótica em adultos estressados e estudantes universitários, demonstrando potencial alternativo de tratamento para estes transtornos e sintomas associados.

Corroborando os achados da meta-análise de Liu, Walsh \& Sheehan (2019) que além de observarem eficácia dos psicobióticos para ansiedade, ressaltaram a escassez de ensaios com amostras psiquiátricas e a prevalência de amostras não clínicas na literatura.

Diante desse cenário de robustez de resultados pré-clínicos significativos, e de ensaios em seres humanos ainda incipientes, Butler et al. (2019) destacam que apesar dos desafios, a ideia de que o tratamento de doenças psiquiátricas pode, no futuro, envolver uma prescrição psicobiótica ou nutricional associada a um psicotrópico tradicional é certamente plausível. O sentimento que se pode considerar mais apropriado no momento, no que diz respeito ao campo do microbioma intestinal e da psiquiatria nutricional, é um otimismo cauteloso, mas justificável.

\section{Conclusão}

Após a análise dos artigos selecionados, verificou-se que a microbiota intestinal pode influenciar significativamente no estado psicológico. Ademais, a maioria das cepas de psicobióticos testadas mostraram-se eficazes, mitigando os sintomas causados pelos quadros de ansiedade, melhorando funções congnitivo-comportamentais, com baixa ocorrência ou ausência de efeitos adversos, o que as direciona como uma nova abordagem terapêutica na atenuação de sintomas de ansiedade e até mesmo para outras comorbidades psiquiátricas, como os transtornos de humor.

Contudo, estudos clínicos com cepas variáveis isoladas ou associadas (pool), e potencialmente psicobióticas no tratamento para ansiedade ainda são escassos. Isto posto, torna-se de suma importância a realização estudos pré-clínicos e clínicos com desenhos metodológicos robustos, visando abranger o conhecimento e aplicação clínica destes adjuvantes na terapêutica de transtornos de ansiedade.

Dessa forma, torna-se essencial o desenvolvimento de pesquisas que objetivem determinar a eficácia e os mecanismos dos psicobióticos, bem como suas possíveis interações com outros medicamentos, e assim subsidiar o seu uso como terapia alternativa nos transtornos de ansiedade.

\section{Referências}

Azpiroz, F., Dubray, C., Bernalier-Donadille, A., Cardot, J. M., Accarino, A., Serra, J., Wagner, A., Respondek, F., \& Dapoigny, M. (2017). Effects of scFOS on the composition of fecal microbiota and anxiety in patients with irritable bowel syndrome: a randomized, double blind, placebo controlled study. Neurogastroenterology and motility : the official journal of the European Gastrointestinal Motility Society, 29(2), 10.1111/nmo.12911.https://doi.org/10.1111/nmo.12911

Bandelow, B., Michaelis, S., \& Wedekind, D. (2017). Treatment of anxiety disorders. Dialogues in clinical neuroscience, 19(2), 93-107. https://doi.org/10.31887/DCNS.2017.19.2/bbandelow

Barros-Santos, T., Silva, K., Libarino-Santos, M., Elisangela Gouveia Cata-Preta, Reis, H. S., Tamura, E. K., de Oliveira-Lima, A. J., Berro, L. F., Uetanabaro, A., \& Marinho, E. (2020). Effects of chronic treatment with new strains of Lactobacillus plantarum on cognitive, anxiety- and depressive-like behaviors in male mice. PloS one, 15(6), e0234037.https://doi.org/10.1371/journal.pone.0234037

Butler, M. I., Mörkl, S., Sandhu, K. V., Cryan, J. F., \& Dinan, T. G. (2019). The Gut Microbiome and Mental Health: What Shou ld We Tell Our Patients?: Le microbiote Intestinal et la Santé Mentale : que Devrions-Nous dire à nos Patients?. Canadian journal of psychiatry. Revue canadienne de psychiatrie, 64(11), 747-760.https://doi.org/10.1177/0706743719874168

Ceppa, F., Mancini, A., \& Tuohy, K. (2019). Current evidence linking diet to gut microbiota and brain development and function. International journal of food sciences and nutrition, 70(1), 1-19.https://doi.org/10.1080/09637486.2018.1462309 
Cheng, L. H., Liu, Y. W., Wu, C. C., Wang, S., \& Tsai, Y. C. (2019). Psychobiotics in mental health, neurodegenerative and neurodevelopmental disorders. Journal of food and drug analysis, 27(3), 632-648.https://doi.org/10.1016/j.jfda.2019.01.002

Colica, C., Avolio, E., Bollero, P., Costa de Miranda, R., Ferraro, S., Sinibaldi Salimei, P., De Lorenzo, A., \& Di Renzo, L. (2017). Evidences of a New Psychobiotic Formulation on Body Composition and Anxiety. Mediators of inflammation, 2017, 5650627.https://doi.org/10.1155/2017/5650627

De Lorenzo, A., Costacurta, M., Merra, G., Gualtieri, P., Cioccoloni, G., Marchetti, M., Varvaras, D., Docimo, R., \& Di Renzo, L. (2017). Can psychobiotics intake modulate psychological profile and body composition of women affected by normal weight obese syndrome and obesity? A double blind randomized clinical trial. Journal of translational medicine, 15(1), 135.https://doi.org/10.1186/s12967-017-1236-2

Depression and Other Common Mental Disorders: Global Health Estimates. Geneva: World Health Organization; 2017. IGO.https://apps.who.int/iris/bitstream/handle/10665/254610/WHO-MSD-MER-2017.2-

eng.pdf;jsessionid=8B95B2C4E7EB2D420509793E86742BD3? sequence $=1$

Dickerson, F., Adamos, M., Katsafanas, E., Khushalani, S., Origoni, A., Savage, C., Schweinfurth, L., Stallings, C., Sweeney, K., Goga, J., \& Yolken, R. H. (2018). Adjunctive probiotic microorganisms to prevent rehospitalization in patients with acute mania: A randomized controlled trial. Bipolar disorders, 20(7), 614-621.https://doi.org/10.1111/bdi.12652

Foster, J. A., Rinaman, L., \& Cryan, J. F. (2017). Stress \& the gut-brain axis: Regulation by the microbiome. Neurobiology of stress, 7, 124136.https://doi.org/10.1016/j.ynstr.2017.03.001

Gualtieri, P., Marchetti, M., Cioccoloni, G., De Lorenzo, A., Romano, L., Cammarano, A., Colica, C., Condò, R., \& Di Renzo, L. (2020). Psychobiotics Regulate the Anxiety Symptoms in Carriers of Allele A of IL-1 $\beta$ Gene: A Randomized, Placebo-Controlled Clinical Trial. Mediators of inflammation, 2020, 2346126.https://doi.org/10.1155/2020/2346126

Hao, Z., Wang, W., Guo, R., \& Liu, H. (2019). Faecalibacterium prausnitzii (ATCC 27766) has preventive and therapeutic effects on chronic unpredictable mild stress-induced depression-like and anxiety-like behavior in rats. Psychoneuroendocrinology, 104, 132-142. https://doi.org/10.1016/j.psyneuen.2019.02.025

He, Z. H., Li, M. D., Liu, C. J., \& Ma, X. Y. (2021). Relationship between body image, anxiety, food-specific inhibitory control, and emotional eating in young women with abdominal obesity: a comparative cross-sectional study. Archives of public health = Archives belges de sante publique, 79(1), 11. https://doi.org/10.1186/s13690-021-00526-2

Herman A. (2019). Probiotics supplementation in prophylaxis and treatment of depressive and anxiety disorders - a review of current research. Zastosowanie suplementacji probiotykami w profilaktyce i leczeniu zaburzeń depresyjnych i lękowych - przegląd dotychczasowych badań. Psychiatria polska, 53(2), 459473.https://doi.org/10.12740/PP/92392

Liu, R. T., Walsh, R., \& Sheehan, A. E. (2019). Prebiotics and probiotics for depression and anxiety: A systematic review and meta-analysis of controlled clinical trials. Neuroscience and biobehavioral reviews, 102, 13-23.https://doi.org/10.1016/j.neubiorev.2019.03.023

Liu, Y. W., Liu, W. H., Wu, C. C., Juan, Y. C., Wu, Y. C., Tsai, H. P., Wang, S., \& Tsai, Y. C. (2016). Psychotropic effects of Lactobacillus plantarum PS128 in early life-stressed and naïve adult mice. Brain research, 1631,1-12.https://doi.org/10.1016/j.brainres.2015.11.018

Mangolini, V. I., Andrade, L. H., Lotufo-Neto, F., \& Wang, Y. P. (2019). Treatment of anxiety disorders in clinical practice: a critical overview of recent systematic evidence. Clinics (Sao Paulo, Brazil), 74, e1316.https://doi.org/10.6061/clinics/2019/e1316

Nash, K., Leota, J., \& Tran, A. (2021). Neural processes in antecedent anxiety modulate risk-taking behavior. Scientific reports, 11(1), 2637.https://doi.org/10.1038/s41598-021-82229-w

Peirce, J. M., \& Alviña, K. (2019). The role of inflammation and the gut microbiome in depression and anxiety. Journal of neuroscience research, 97(10), 1223-1241.https://doi.org/10.1002/jnr.24476

Sharma, R., Gupta, D., Mehrotra, R., \& Mago, P. (2021). Psychobiotics: The Next-Generation Probiotics for the Brain. Current microbiology, 78(2), 449463.https://doi.org/10.1007/s00284-020-02289-5

Slee, A., Nazareth, I., Bondaronek, P., Liu, Y., Cheng, Z., \& Freemantle, N. (2019). Pharmacological treatments for generalised anxiety disorder: a systematic review and network meta-analysis. Lancet (London, England), 393(10173), 768-777.https://doi.org/10.1016/S0140-6736(18)31793-8

Smith, K. S., Greene, M. W., Babu, J. R., \& Frugé, A. D. (2019). Psychobiotics as treatment for anxiety, depression, and related symptoms: a systematic review. Nutritional neuroscience, 1-15. Advance online publication.https://doi.org/10.1080/1028415X.2019.1701220

Souza, M. T.; Silva, M. D.; Carvalho, R. Revisão integrativa: o que é e como fazer. Einstein, v. 8, p. 102-106, 2010.

Steenbergen, L., Sellaro, R., van Hemert, S., Bosch, J. A., \& Colzato, L. S. (2015). A randomized controlled trial to test the effect of multispecies probiotics on cognitive reactivity to sad mood. Brain, behavior, and immunity, 48, 258-264.https://doi.org/10.1016/j.bbi.2015.04.003

Yang, X. Y., Yang, N. B., Huang, F. F., Ren, S., \& Li, Z. J. (2021). Effectiveness of acupuncture on anxiety disorder: a systematic review and meta-analysis of randomised controlled trials. Annals of general psychiatry, 20(1), 9.https://doi.org/10.1186/s12991-021-00327-5

Yunes, R. A., Poluektova, E. U., Vasileva, E. V., Odorskaya, M. V., Marsova, M. V., Kovalev, G. I., \& Danilenko, V. N. (2020). A Multi-strain Potential Probiotic Formulation of GABA-Producing Lactobacillus plantarum 90sk and Bifidobacterium adolescentis 150 with Antidepressant Effects. Probiotics and antimicrobial proteins, 12(3), 973-979. https://doi.org/10.1007/s12602-019-09601-1 